\title{
Effects of Daily Events on Mood States in Major Depressive Disorder
}

\author{
Frenk Peeters and Nancy A. Nicolson \\ Maastricht University
}

\author{
Johannes Berkhof \\ University of Leuven
}

\author{
Philippe Delespaul and Marten deVries \\ Maastricht University
}

\begin{abstract}
Major depressive disorder (MDD) is characterized by high negative affect (NA) and low positive affect (PA), but little is known about emotional reactivity in daily life. The authors used experience sampling methodology to investigate changes in NA and PA following minor daily events in MDD compared with healthy participants. Contrary to expectation, MDD participants did not report more frequent negative events, although they did report fewer positive events. Multilevel regression showed that both NA and PA responses to negative events were blunted in the MDD group, whereas responses to positive events were enhanced. NA responses to negative events persisted longer in MDD participants. Depressed participants with a positive family history or longer current episodes showed relatively greater NA responses to negative events.
\end{abstract}

Investigations of the dimensionality of mood states have provided evidence for the existence of relatively independent negative and positive affective states (Diener \& Emmons, 1984; Watson, 2000). The dimension of negative affect (NA) represents the extent to which individuals experience feelings such as nervousness, tension, irritation, and guilt. Positive affect (PA) reflects the level of pleasurable engagement with one's environment, characterized by feelings such as enthusiasm, interest, and satisfaction. Previous studies have shown that the symptomatic phase of an episode of major depressive disorder (MDD) is characterized by high levels of NA and, more specifically, low levels of PA (Clark, Beck, \& Stewart, 1990; Jolly, Murray, Kramer, \& Wherry, 1994; Watson et al., 1995). However, the dynamics of these mood states in MDD are not well understood.

Minor events in daily life are known to induce momentary changes in NA and PA. Negative events lead to increases in NA (Gable, Reis, \& Elliot, 2000; Marco, Neale, Schwartz, Shiffman, \& Stone, 1999; Suls, Green, \& Hillis, 1998; van Eck, Nicolson, \& Berkhof, 1998), whereas their effect on PA is generally modest (David, Green, Martin, \& Suls, 1997; Gable et al., 2000; van Eck et al., 1998). Changes in PA are more strongly related to the

Frenk Peeters, Nancy A. Nicolson, Philippe Delespaul, and Marten deVries, Department of Psychiatry and Neuropsychology, Maastricht University, Maastricht, the Netherlands; Johannes Berkhof, Department of Psychology, University of Leuven, Leuven, Belgium.

Johannes Berkhof is now at the Department of Clinical Epidemiology and Biostatistics, Free University Medical Center, Amsterdam, the Netherlands.

We thank Lilly Finders, Truda Driessen, and Frieda Van Goethem for research assistance.

Correspondence concerning this article should be addressed to Frenk Peeters, who is now at the Department of Psychiatry, Academic Hospital Maastricht, P.O. Box 5800, 6202 AZ Maastricht, the Netherlands. E-mail: f.peeters@sp.unimaas.nl occurrence of positive than negative minor events (David et al., 1997; Gable et al., 2000; Langston, 1994). The fact that low PA appears to be a specific feature of MDD makes a better understanding of factors that influence this mood dimension important. Research investigating responses to both negative and positive events could therefore add to our knowledge about mood disturbances in the daily life of depressed people.

The interplay between minor events and mood states during episodes of MDD is important for a number of reasons. First, fluctuations in mood are a common but poorly understood phenomenon in people with MDD. As in healthy people, it can be assumed that mood states of clinically depressed individuals are influenced by daily negative and positive events. Second, changes in neuroendocrine and immunological systems are thought to be important elements of the pathophysiology of MDD (Connor \& Leonard, 1998; Holsboer, 2000). Because these physiological systems are activated by emotional responses to stress (Segerstrom, Taylor, Kemeny, \& Fahey, 1998; van Eck, Berkhof, Nicolson, \& Sulon, 1996), information about mood responses to daily events can fill some gaps in theoretical models linking stress to MDD. Third, cognitive-behavioral treatment for MDD is based, at least in part, on the assumption that changes in daily behavior and cognitive appraisal of daily events are ingredients of a successful therapy (Beck, Rush, Shaw, \& Emery, 1979; Jacobson et al., 1996). Knowledge about the interplay between minor daily events and mood can contribute to a better understanding of mechanisms underlying these assumptions.

Daily process designs, such as the experience sampling method (ESM), have been shown to be valid and reliable techniques for the simultaneous assessment of mood, behaviors, and cognitions in the natural environment (Csikszentmihalyi \& Larson, 1987; Delespaul, 1995; deVries, 1992; Marco et al., 1999; Swendsen et al., 2000; van Eck et al., 1998). Events and mood are measured at frequent intervals during the day, which allows the study of tem- 
poral relationships between events and mood states that cannot be captured by end-of-day reports. The difficulties that many people have in accurately detecting contingencies between events and mood are also bypassed. Furthermore, ESM reduces bias in recall, which is especially relevant in research on mood disorders (Morris, 1999).

The present study used ESM to examine momentary mood changes following naturally occurring negative and positive events in the daily life of clinically depressed and healthy people. Previous research has reported larger NA responses to negative daily events in neurotic or distressed individuals (Suls et al., 1998; Swendsen, 1998; van Eck et al., 1998). A larger decrease in PA in response to negative events was found in individuals who reported greater perceived stress (van Eck et al., 1998). Given the absence of comparable studies in clinically depressed individuals and uncertainty about the generalizability of results from studies in "analogue" populations (Coyne, 1994; Flett, Vredenburg, \& Krames, 1997; Santor \& Coyne, 2001), we made no a priori hypotheses concerning the direction of differences in mood reactivity between clinically depressed and healthy people. We expected the average levels of NA to be high in depressed participants and PA to be low, in comparison with healthy participants. We predicted that NA would increase and PA would decrease following negative events in both depressed and healthy participants. Following positive events, we predicted a decrease in NA and an increase in PA.

Within the framework of transactional stress theory, how an event is appraised is an important determinant of its impact on mood (Lazarus \& Folkman, 1984). Primary appraisals such as unpleasantness and stressfulness of negative events are associated with larger changes in mood (Marco et al., 1999; van Eck et al., 1998). Expecting that depressed individuals would appraise events differently than healthy individuals (Beck et al., 1979), we predicted that these subjective evaluations would be significant determinants of differences in mood reactivity between depressed and healthy participants. Previous studies have reported that the effects of minor events on mood are generally transient but may be longer lasting in anxious or stressed individuals (Bolger \& Zuckerman, 1995; van Eck et al., 1998). In other words, mood recovery is delayed. We therefore examined differences in the persisting effects of prior events on mood states between depressed and healthy participants.

To gain broader insight into possible mechanisms, we investigated the effects of clinical characteristics on mood reactivity within the group of depressed participants. First, we hypothesized that severity of episode would influence mood reactivity to daily events. Second, longer duration of the current episode and a history of previous depressive episodes have been associated with an increased vulnerability to psychosocial stressors (Kendler, Thornton, \& Gardner, 2000), changes in self-concept and coping strategies (Coyne, Gallo, Klinkman, \& Clarko, 1998), and increased use of dysfunctional cognitive styles (Segal, Williams, Teasdale, \& Gemar, 1996). We therefore hypothesized that duration of the current episode and a history of previous episodes of MDD would increase reactivity in NA and PA to negative events and decrease reactivity of both mood states to positive events. Third, given evidence that genetic vulnerability for mood disorders may express itself in heightened neuroticism as well as in alterations in regulation of the hypothalamus-pituitary-adrenal (HPA) axis (Krieg, Lauer, Schreiber, Modell, \& Holsboer, 2001; Maier,
Lichtermann, Minges, \& Heun, 1992), we expected that depressed participants with a family history for mood disorders might show greater affective responses to negative events. Finally, many depressed individuals show a diurnal pattern in symptom severity with some alleviation during the course of the day (American Psychiatric Association, 1994). We therefore tested whether effects of events on mood states were influenced by time of their occurrence.

\section{Method}

\section{Participant Recruitment and Selection}

Forty-seven participants diagnosed with MDD were recruited among individuals seeking treatment at the community mental health center and the outpatient clinic of the local psychiatric hospital in Maastricht, the Netherlands. The main inclusion criterion was a primary diagnosis of MDD, as assessed with the Structured Clinical Interview for DSM-IV (SCID; First, Spitzer, Gibbon, \& Williams, 1995) by a research psychiatrist (Frenk Peeters). Entry was restricted to individuals 18-65 years of age with a 17-item Hamilton Depression Rating Scale (HDRS; Hamilton, 1967) score of $\geq 18$, indicating at least moderate severity of MDD. Exclusion criteria were current substance abuse or psychotic symptoms (both assessed with the SCID) and insufficient fluency in Dutch. None of the participants was using antidepressants, but use of low-dose anxiolytic drugs was allowed during the study. One depressed participant was excluded from the analysis because of inadequate compliance with the research protocol (see Procedure).

Thirty-nine healthy participants, matched as a group to the patient sample for gender and age, were recruited from available research pools, through staff from academic affiliations, and through an advertisement in a local newspaper. Additional exclusion criteria for the healthy participants were a lifetime history of any Diagnostic and Statistical Manual of Mental Disorders (4th ed., DSM-IV; American Psychiatric Association, 1994) Axis I disorder (assessed with the initial screening section of the SCID) or any inpatient treatment for an Axis I psychiatric disorder in a first-degree relative. Of the 55 potential controls who were screened, 16 were excluded: 8 for having a history of psychiatric disorder, 3 for inpatient psychiatric treatment in a first-degree relative, 3 for having somatic disorders that could affect the HPA axis (an exclusion criterion for cortisol measures in the same study), and 2 for other reasons (e.g., hearing impairment). The study was approved by the local medical ethics committee, and written informed consent was obtained from all participants. Participants were paid $\$ 30$ for completing the study.

The final sample consisted of 46 depressed and 39 healthy participants Demographic characteristics and Symptom Checklist-90 scores (Derogatis, Lipman, \& Cori, 1973) for the two groups are presented in Table 1. In the depressed group, $67 \%$ were married or living with a partner, compared with $76 \%$ in the healthy group, $\chi^{2}(1, N=85)=0.95, p=.33$. Educational level (dichotomized as at least high school completed or not) was higher in the healthy than in the depressed group ( $89 \%$ vs. $56 \%$ high school completers $), \chi^{2}(1, N=85)=11.49, p<.01$. Employment status differed, $\chi^{2}(1$, $N=85)=15.53, p<.01$, with only $36 \%$ of participants with MDD currently working (compared with $79 \%$ of healthy participants).

MDD participants completed the Beck Depression Inventory (BDI; Beck, Ward, \& Mendelson, 1961), a self-report measure of symptom severity. Information on length of the current depressive episode was obtained during the SCID interview. Family history for mood disorders in first-degree relatives (treated or untreated) was obtained in an unstructured interview on the basis of a genogram. Clinical characteristics of the MDD participants are summarized in Table 1 . In addition to the primary diagnosis of MDD, 21 patients (46\%) had a secondary Axis I diagnosis, as follows: generalized social phobia $(n=9)$, dysthymic disorder $(n=5)$, panic disorder with agoraphobia $(n=4)$, posttraumatic stress disorder $(n=$ 
Table 1

Demographic and Clinical Characteristics of Depressed and Healthy Participants

\begin{tabular}{lccc}
\hline \multicolumn{1}{c}{ Variable } & Participants & \\
\cline { 2 - 3 }$n$ & MDD & Healthy & $p$ \\
\hline Men & 46 & 39 & \\
$\quad$ Women & 20 & 16 & \\
Age in years & 26 & 23 & .09 \\
SCL-90 total score & $259.8(53.9)$ & $109.8(21.8)$ & $<.001$ \\
SCL-90 depression & $57.1(11.6)$ & $18.9(4.1)$ & $<.001$ \\
HDRS & $23.9(3.9)$ & & \\
BDI & $28.4(9.8)$ & & \\
Duration current & & & \\
$\quad$ episode in months & 20.3 (range 2-118) & \\
Previous episode(s) & $20(44 \%)$ & \\
Positive family & & & \\
$\quad$ history & $25(54 \%)$ & & \\
\hline
\end{tabular}

Note. Means (and standard deviations) shown for continuous variables. Means were compared using $t$ tests; categorical variables were compared with the use of chi-square tests. MDD = major depressive disorder; SCL-90 $=$ Symptom Checklist-90; HDRS $=17$-item Hamilton Depression Rating Scale; BDI = Beck Depression Inventory.

4), specific phobia $(n=4)$, and bulimia nervosa $(n=1)$. Of these, four MDD participants had three or more Axis I diagnoses.

\section{Procedure}

ESM was used to collect data from participants at selected moments during their daily activities. Participants received auditory signals (beeps) from a wristwatch programmed to emit 10 beeps between 7:30 a.m. and 10:30 p.m. each day, at semirandom intervals of approximately $90 \mathrm{~min}$. After receiving a beep, participants completed self-report forms concerning current mood, negative and positive events, and their appraisals. Participants completed ESM reports for 6 consecutive days, including a weekend. During a briefing session, study aims and procedures were explained. In a final session, the ESM booklets were checked for legibility and missing data.

Compliance with the procedure was generally good. The criteria set for inclusion in the analyses (more than 20 ESM reports completed within 25 min after the programmed time of the beep) were met by all participants except one depressed participant. Participants included in the analyses completed an average of $85 \%$ of all possible responses within the time limit, resulting in an average number of valid responses of 50.7 per participant. Mean number of valid responses was somewhat higher in the healthy than in the depressed group (53.2 vs. 48.6), $t(84)=3.3, p=.003$.

\section{Measures}

Mood assessment. Momentary mood states were assessed with 16 adjectives rated on 7 -point scales $(1=$ not at all, $7=$ very $)$. Factor analyses (principal-components analysis with varimax rotation) on mean scores aggregated per subject and on within-subject $z$ scores both identified two mood factors with eigenvalues greater than 1 . These factors accounted for $81.1 \%$ of the total variance in subject mean scores and $46.1 \%$ of the variance in within-subject $z$ scores. Ratings on the items anxious, irritated, restless, tense, guilty, irritable, easily distracted, and agitated were averaged to form a NA scale (Cronbach's alpha $=.91$, based on all 4,535 ESM reports; item loadings on Factor 1 ranged between .66 and .88 , loadings on Factor 2, between -.16 and -.40). Ratings on the items energetic, enthu- siastic, happy, cheerful, talkative, strong, satisfied, and self-assured were averaged to form a PA scale $(\alpha=.95$; item loadings on Factor 1 ranged between -.08 and -.42 , loadings on Factor 2, between .73 and .92 ). The average NA level (aggregated over participant means) was $1.3(S D=0.3)$ in healthy participants and $2.9(S D=0.9)$ in depressed participants. Average PA levels were $4.5(S D=0.9)$ and $2.1(S D=0.7)$ for healthy and depressed participants, respectively. Correlations between NA and PA, based on raw data, were $-.19(p<.01)$ for depressed and $-.33(p<.01)$ for healthy participants.

Stressful events. At each beep, participants were asked briefly to describe any positive and/or negative event that may have taken place since the last ESM report. Although participants were instructed to report only events or situations that actually took place in their daily environment in the preceding interval, some event reports clearly referred to internal states only (e.g., current ruminations about past events, personal health concerns). Following preestablished criteria, the research team identified such internal events by consensus. Reports of internal events were not used in the analysis, to avoid confounding of event and mood measures. In the depressed group, $16 \%$ of all event reports referred to internal states compared with $8 \%$ in the healthy group. We identified events as prior when the previous ESM report on the same day $(t-1)$ mentioned an event, to address persisting effects on mood states in the analysis.

Appraisals. On 7 -point scales $(1=$ not at all, $7=$ very $)$, participants rated positive events on the dimensions pleasant, important, and stressful; negative events were rated on the dimensions unpleasant, important, and stressful. Correlations, over all ESM reports from all participants, between the appraisals of positive events were low to moderate (pleasant with important: $r=.49, p<.01$; pleasant with stressful: $r=-.25, p<.01$; important with stressful: $r=.01, p=.60$ ). The correlations between appraisals of negative events were moderate to strong (unpleasant with important: $r=.30, p<.01$; unpleasant with stressful: $r=.55, p<.01$; important with stressful: $r=.32, p<.01$ ).

\section{Statistical Model}

Because the experience sampling observations are nested within days within participants, we estimated the effects of daily events, depression, and individual characteristics on mood states with multilevel or hierarchical linear analysis, which is a variant of multiple regression appropriate for nested data. Separate models for NA and PA were estimated using the program MLwiN (Goldstein et al., 1998).

The regression equation can be expressed as follows:

$$
(\text { Mood })_{i j t}=b_{0 m}+\sum_{q=1}^{Q} b_{q m} x_{q i j t}+r_{i j t},
$$

where $(\operatorname{Mood})_{i j t}$ is the NA or PA score of participant $i$ at beep $t$ on day $j$. The intercept is denoted by $b_{0 m}, x_{q i j t}$ is an entry of explanatory variable $x_{q}$, $b_{q m}$ is the corresponding regression coefficient, and $r_{i j t}$ is an error term. The index $m$ indicates whether the participant is depressed $(m=1)$ or healthy $(m=0)$. By indexing all regression coefficients with the label $m$, we estimated all effects separately for the depressed and healthy groups. The error term is itself a function of error terms that capture the dependencies among the mood observations. Such dependencies occur because two observations tend to be more similar if (a) taken at points closer in time on the same day, (b) taken on the same day rather than on two different days, and (c) taken from one participant rather than from different participants. To account for these sources of dependency, we (a) modeled the autocorrelation between two subsequent observations as an exponentially decaying function of the time interval between these observations, (b) estimated day level variance to account for day-to-day variability, and (c) modeled a random intercept to account for interindividual variability in mood levels. Ignoring these dependencies biases the estimated standard errors of the regression coefficients and may lead to incorrect inferences about the 
effects of the explanatory variables. It should be noted that autocorrelation controls for the influence of the previous mood score on the current mood score (after having adjusted for the level of the covariates).

The following dummy-coded ( 0 or 1$)$ variables were predictors in the model: PosEvent ( 1 if a positive event was reported), NegEvent (1 if a negative event was reported), PriorPosEvent ( 1 if a positive event was reported at the previous beep), and PriorNegEvent (1 if a negative event was reported at the previous beep). The values of PriorPosEvent and PriorNegEvent were set to 0 at the first beep of the day. We also estimated the effects of a positive or negative event at the last beep of the day on the mood scores at the first beep of the next day. These effects were negligible and were therefore excluded from the model.

Additional explanatory variables were either centered around the grand mean or effect coded $(-1,1$ instead of 0,1$)$ so that inclusion in the model would not automatically change the previously estimated effects of events on mood. The event appraisal scores were centered around the grand mean or were set to zero if no event was reported. To examine the effect of gender on the relation between depression, events, and mood states, we included the variable gender ( -1 if female, 1 if male), as well as its interactions with PosEvent, NegEvent, PriorPosEvent, and PriorNegEvent.

To assess the impact of clinical characteristics in MDD on mood responses, we included the following variables in the model: severity of depression (BDI score centered around mean), duration of current depressive episode (in months, centered around mean), episode (1 if previous episode; -1 if first episode), and family history for mood disorders ( 1 if positive history; -1 if negative history). Because these clinical variables can have an effect in the MDD group only, they were modeled as interactions with group; as a result, scores for healthy participants take the value 0 . Interactions between clinical characteristics and events (PosEvent, NegEvent, PriorPosEvent, and PriorNegEvent) were also estimated.

Finally, we modeled the diurnal pattern in mood states with a linear function. The time variable was centered around the midpoint of the sampling times (3:06 p.m.) and included as a covariate in the model.

Significance of the regression coefficients was tested by dividing the estimated effects by their standard errors. These ratios are approximately normally distributed. Two-tailed tests were used even when hypotheses were directional. Significance levels were set at alpha $=.05$.
Results

\section{Frequency and Appraisals of Daily Events}

Group differences in frequencies and appraisals of events are presented in Table 2. In total, 1,762 events were reported during the 6 days. Four participants ( 2 in each group) reported no events. The frequency of negative events was similar in both groups, but the depressed group reported fewer positive events. Negative events were appraised as more unpleasant, more important, and more stressful by the depressed than by the healthy participants. Positive events were appraised as less pleasant, equally important, and more stressful by the depressed participants.

\section{Effects of Events on Mood States}

We present the results of the multilevel analyses for changes in NA and PA separately in table format. In each table, the effects of negative and positive events on mood states are shown for depressed and healthy participants; the last column shows the differences between the groups. Because event and mood reports were collected simultaneously, it is important to note that significant effects in the multilevel model cannot firmly establish a causal relationship between event occurrence and subsequent changes in mood.

NA. Basal NA levels were higher $(\beta=2.834, S E=.097$, vs. $\beta=1.220, S E=.141)$ in depressed than in healthy participants (difference: $\beta=1.614, S E=.176, z=9.17, p<.01$ ). NA was further elevated in more severely depressed participants $(\beta=.032$, $S E=.010, z=3.20, p<.01)$ and in depressed participants with episodes of longer duration $(\beta=.012, S E=.004, z=3.00, p<$ $.01)$. Neither a previous episode nor a positive family history was associated with additional increases in NA levels.

In both groups, negative events led to increased NA (see left half of Table 3). Although levels of unpleasantness (valence) and importance had no additional effects on NA reactivity, events appraised as more stressful further increased NA. Depressed par-

Table 2

Mean Frequencies (and Standard Deviations) and Appraisal Ratings of Daily Events in Depressed $(n=44)$ and Healthy $(n=37)$ Participants

\begin{tabular}{|c|c|c|c|c|c|c|}
\hline \multirow[b]{3}{*}{ Variable } & \multicolumn{4}{|c|}{ Participants } & \multirow[b]{3}{*}{$|t(79)|$} & \multirow[b]{3}{*}{$p$} \\
\hline & \multicolumn{2}{|c|}{ MDD } & \multicolumn{2}{|c|}{ Healthy } & & \\
\hline & $M$ & $S D$ & $M$ & $S D$ & & \\
\hline Frequency of negative events & $16 \%$ & 12 & $17 \%$ & 13 & 0.35 & .7 \\
\hline Unpleasant & 5.6 & 1.0 & 4.7 & 0.8 & 4.33 & $<.001$ \\
\hline Important & 4.6 & 1.4 & 3.9 & 1.2 & 2.18 & .03 \\
\hline Stressful & 5.4 & 1.3 & 3.3 & 1.2 & 7.27 & $<.001$ \\
\hline Frequency of positive events & $21 \%$ & 16 & $34 \%$ & 24 & 3.03 & .003 \\
\hline Pleasant & 5.3 & 1.0 & 5.8 & 0.9 & 2.35 & .02 \\
\hline Important & 5.3 & 1.3 & 5.1 & 1.0 & 0.58 & .56 \\
\hline Stressful & 2.4 & 1.3 & 1.6 & 0.8 & 3.36 & $<.001$ \\
\hline $\begin{array}{l}\text { Frequency of reports of negative and positive } \\
\text { events within same time interval }\end{array}$ & $8 \%$ & 9 & $13 \%$ & 13 & $1.26^{\mathrm{a}}$ & .21 \\
\hline
\end{tabular}

Note. Frequencies of events are expressed as percentage of total beeps in which an event was reported. MDD = major depressive disorder.

${ }^{\mathrm{a}} d f=38$. 
Table 3

Multilevel Model Estimates of Effects of Events on Negative Affect

\begin{tabular}{|c|c|c|c|c|c|c|}
\hline \multirow[b]{2}{*}{ Variable } & \multicolumn{3}{|c|}{ Negative event $\beta$ (SE) } & \multicolumn{3}{|c|}{ Positive event $\beta$ (SE) } \\
\hline & $\begin{array}{c}\text { MDD } \\
\text { participants }\end{array}$ & $\begin{array}{c}\text { Healthy } \\
\text { participants }\end{array}$ & Difference & $\begin{array}{c}\text { MDD } \\
\text { participants }\end{array}$ & $\begin{array}{l}\text { Healthy } \\
\text { participants }\end{array}$ & Difference \\
\hline Event & $.449(.056) * * *$ & $.678(.066)^{* * *}$ & $-.229(.087)^{* *}$ & $-.221(.045)^{* * *}$ & $-.020(.052)$ & $-.201(.069)^{* *}$ \\
\hline Valence & $.040(.029)$ & $-.006(.028)$ & $-.046(.041)$ & $.035(.026)$ & $-.015(.023)$ & $.049(.035)$ \\
\hline Importance & $-.020(.018)$ & $-.009(.018)$ & $-.011(.026)$ & $-.076(.022)^{* * *}$ & $.019(.018)$ & $-.095(.028)^{* * *}$ \\
\hline Stressfulness & $.173(.025)^{* * *}$ & $.201(.024) * * *$ & $-.028(.035)$ & $.118(.018) * * *$ & $.060(.022)^{* *}$ & $.057(.028)^{*}$ \\
\hline Prior event & $.187(.044) * * *$ & $.019(.056)$ & $.167(.073) *$ & $-.039(.039)$ & $-.001(.049)$ & $-.038(.064)$ \\
\hline
\end{tabular}

Note. The analysis is based on 4,306 beeps nested within 85 persons. Valence of event is unpleasant for negative event and pleasant for positive event. MDD = major depressive disorder.

$* p<.05 . \quad * * p<.01 . \quad * * * p<.001$.

ticipants showed significantly smaller increases in NA in response to negative events than healthy participants. Prior negative events, on the other hand, had a persistent effect in MDD only.

In the healthy group, positive events only minimally decreased NA; positive events appraised as relatively stressful resulted in a slight increase in NA (see right half of Table 3). In comparison, depressed participants showed a more pronounced decrease in NA in response to positive events, particularly in the case of events appraised as relatively important. In the MDD group, stressful positive events were followed by a significantly greater increase in NA than observed in the healthy group. Prior positive events had no persistent effect on NA in either group.

In contrast to results for the healthy group, depressed women showed higher basal NA than depressed men $(\beta=.264, S E=$ $.099, z=2.67, p<.01$ ). In addition, depressed women responded with larger decreases in NA following positive events than depressed men $(\beta=-.143, S E=.039, z=3.67, p<.01)$. Other gender effects in depressed and healthy participants were nonsignificant.

We also tested for the effects of interactions between clinical characteristics (severity of depression, duration of the current episode, previous episode, positive family history) and daily events on NA within the depressed group. NA responses to negative events were further elevated in depressed participants with longer current episodes of depression $(\beta=.004, S E=.002$, $z=2.32, p=.02)$ and in those with a positive family history $(\beta=$
$.111, S E=.043, z=2.58, p=.01)$. Depression severity and previous episodes had no significant influence on NA reactivity to negative events. For NA reactivity, we found no significant effects of interactions between clinical characteristics and positive events. Clinical characteristics showed no influence on NA reactivity to prior events, either negative or positive.

Within the depressed group, NA levels declined significantly over the day $(\beta=-.031, S E=.004, z=7.42, p<.01)$. Increases in NA following negative events remained unchanged, but decreases in NA following positive events were slightly smaller later in the day $(\beta=.024, S E=.009, z=2.60, p<.01)$.

Positive affect. Basal PA levels were lower $(\beta=2.001, S E=$ .112 , vs. $\beta=4.447, S E=.162$ ) in depressed than in healthy participants (difference: $\beta=2.447, S E=.202, z=12.11, p<$ $.01)$, with more severely depressed participants having even lower levels $(\beta=-.029, S E=.012, z=2.41, p=.02)$. Other clinical characteristics showed no significant influence on basal PA levels.

Negative events decreased PA in all participants (see left half of Table 4), but these decreases were significantly smaller in depressed participants. Negative events that were appraised as more stressful were followed by further decreases in PA in healthy participants only. Prior negative events had no persisting effect on PA in either group.

Current and prior positive events were associated with increases in PA (see right half of Table 4). However, positive events resulted in larger increases in PA in depressed than in healthy participants.

Table 4

Multilevel Model Estimates for Effects of Events on Positive Affect

\begin{tabular}{|c|c|c|c|c|c|c|}
\hline \multirow[b]{2}{*}{ Variable } & \multicolumn{3}{|c|}{ Negative event $\beta$ (SE) } & \multicolumn{3}{|c|}{ Positive event $\beta$ (SE) } \\
\hline & $\begin{array}{c}\text { MDD } \\
\text { participants }\end{array}$ & $\begin{array}{c}\text { Healthy } \\
\text { participants }\end{array}$ & Difference & $\begin{array}{c}\text { MDD } \\
\text { participants }\end{array}$ & $\begin{array}{l}\text { Healthy } \\
\text { participants }\end{array}$ & Difference \\
\hline Event & $-.117(.059)^{*}$ & $-.356(.071)^{* * *}$ & $.238(.093)^{* *}$ & $.483(.048)^{* * *}$ & $.308(.056) * * *$ & $.175(.074)^{*}$ \\
\hline Valence & $-.058(.032)$ & $-.008(.029)$ & $-.051(.043)$ & $.051(.027)$ & $.056(.025)^{*}$ & $-.005(.037)$ \\
\hline Importance & $.022(.019)$ & $.009(.019)$ & $.013(.027)$ & $.007(.023)$ & $.029(.019)$ & $.023(.029)$ \\
\hline Stressfulness & $.014(.026)$ & $-.105(.026)^{* * *}$ & $.119(.037)^{* * *}$ & $-.027(.019)$ & $-.029(.024)$ & $.001(.030)$ \\
\hline Prior event & $-.013(.047)$ & $.069(.061)$ & $-.082(.078)$ & $.160(.042)^{* * *}$ & $.179(.052) * * *$ & $-.019(.069)$ \\
\hline
\end{tabular}

Note. The analysis is based on 4,306 beeps nested within 85 persons. Valence of event is unpleasant for negative event and pleasant for positive event. MDD = major depressive disorder

$* p<.05 . \quad * * p<.01 . \quad * * * p<.001$. 
Current events that were appraised as more pleasant were followed by further significant PA increases in healthy participants and a similar trend in depressed participants. In contrast to the findings for negative events, stressfulness of positive events had no effect on PA for either healthy or depressed participants. None of the results for PA were influenced by gender.

In contrast to the results for NA, there were no significant interaction effects between clinical characteristics and a current event on PA. However, prior negative events led to a persistent lowering of PA in depressed participants who had experienced a previous episode $(\beta=-.095, S E=.045, z=2.11, p=.04)$. PA levels increased slightly over the course of the day in depressed participants $(\beta=.024, S E=.005, z=5.07, p<.01)$, but PA reactivity following negative and positive events showed no diurnal pattern.

The finding that MDD participants reported relatively few positive events raises the question of whether individual differences in event frequency may have influenced mood responses to events. To address this question, we added person-level mean frequencies for both positive and negative events as explanatory variables in the multilevel model. Event frequencies had no significant effect on either NA levels or NA reactivity to events. For PA, event frequencies had no effects on mood levels; individuals (in particular, healthy participants) who reported relatively many positive events showed marginally larger PA responses to these events. Differences, however, between MDD and healthy groups in the effects of events (both positive and negative) on PA remained significant.

Finally, because few MDD participants were currently working, differences between the two groups in daily activities might have resulted in exposure to different sorts of events. If so, one might expect to find larger weekday versus weekend effects on mood reactivity in the healthy group. We therefore examined weekendweekday patterns of mood and mood reactivity in the multilevel model. MDD participants showed a slight increase in PA levels on the weekend, but we found no evidence that weekends had any effect on either PA or NA reactivity (data from this analysis are not shown).

\section{Discussion}

To our knowledge, this is the first study of the influence of negative and positive minor events on mood states in clinically depressed and healthy individuals in their natural environment. Some of the findings are surprising or counterintuitive. For example, MDD participants did not report more frequent negative events, although they did report fewer positive events and appraised both types of events as more stressful. Mood responses to negative events were more pronounced in the healthy participants, whereas mood responses to positive events were larger in depressed participants. Events experienced as more stressful influenced the magnitude of the responses of NA and PA and contributed further to the different effects on mood reactivity in the two groups. Within the MDD group, duration of current episode and a positive family history for mood disorders enhanced NA responses to negative events, but responses remained blunted when compared with healthy participants.

The finding that healthy and depressed participants reported similar numbers of negative events contradicts results of most previous studies. Using retrospective research designs, these studies found more frequent negative daily events in people with MDD (Grosscup \& Lewinsohn, 1980; Lewinsohn, 1975; Lewinsohn \& Amenson, 1978; Ravindran, Griffiths, Waddell, \& Anisman, 1995; for an exception, see Coyne, Aldwin, \& Lazarus, 1981). Given the methodological limitations inherent in retrospective designs, especially in people diagnosed with MDD, our results may provide a more accurate picture of negative event frequency in MDD. The fact that we explicitly drew a distinction between external and internal events to avoid confounding of event and disorder contributes to the robustness of our finding. Consistent with the previous studies, depressed participants reported fewer positive events than healthy participants. According to Gable et al. (2000), positive events are more likely to be actively initiated by an individual, whereas negative events are more likely to happen spontaneously in daily life. In line with this hypothesis, symptoms such as lack of energy and anhedonia in MDD could reduce the likelihood of positive event occurrence, as we found. Taken together, our findings underscore the need to assess both negative and positive events in daily life in order to obtain the best indicator of the effects of depressive symptomatology on well-being (Holahan, Moos, Holahan, \& Cronkite, 2000; Kanner, Coyne, Schaefer, \& Lazarus, 1981).

Negative events were followed by increases in NA and decreases in PA; as in previous studies (Gable et al., 2000; Marco et al., 1999; Suls et al., 1998; van Eck et al., 1998), these changes were enhanced when the negative event was appraised as stressful. Surprisingly, although depressed participants experienced negative events as more unpleasant, important, and stressful, their mood responses were blunted in comparison to healthy participants. On the one hand (event appraisals), these results can be interpreted as evidence for environmental hypersensitivity; on the other hand (mood changes), they may be construed as evidence for hyposensitivity. Objective aspects of the events and their timing might help reconcile the two interpretations, but these details cannot be obtained in a naturalistic study. It has been argued that a strong and immediate response to negative events (including physiological, affective, cognitive, and behavioral changes), followed by a rapid return to homeostasis, is beneficial for the organism in both the short and the long term (Taylor, 1991). Given the characteristic fatigue, cognitive dysfunctions, and motivational problems in MDD, it is plausible that individuals suffering from MDD respond less strongly to negative events but recover more slowly. The observed reduced mood reactivity to negative events and a more persistent effect of prior negative events on NA in MDD are consistent with this hypothesis.

Could the current findings on mood reactivity be due to ceiling or floor effects? Given that mean NA level in depressed participants was only 2.8 on a 7-point scale, a ceiling effect for NA responses in MDD seems unlikely. PA responses in MDD might potentially be blunted because of a floor effect, as mean PA was very low in this group (2.0 on a 7-point scale). However, because most appraisals of negative events moderated PA responses similarly in both groups, a floor effect seems unlikely. In addition, depressed participants with more severe symptoms displayed lower mean PA levels but did not exhibit a greater blunting of PA response than participants with less severe symptoms. It remains possible that low mean NA levels in the healthy group reduced the likelihood of further decreases and that this floor effect artificially 
inflated the observed difference between the two groups in NA reactivity to positive events. Ceiling effects for PA in the healthy participants are less probable, as their mean PA level was only 4.5 on a 7-point scale.

The literature on mood changes in daily life is dominated by studies of daily hassles. The current results indicate that daily uplifts are also important determinants of mood, in both depressed and healthy people. As in a previous study (David et al., 1997), the effects of positive events on mood states were smaller than the effects of negative events. Compared with healthy participants, depressed participants appraised positive events as less pleasant and as more stressful but as equally important. In contrast to the findings for negative events, the participants with MDD showed greater mood reactivity than controls, with larger decreases in NA and increases in PA. More stressful positive events resulted in a larger increase in NA in the depressed group, whereas more important positive events led to a further decrease in NA. Previous research has shown that intense PA is often preceded by periods of low PA (Diener, Colvin, Pavot, \& Allman, 1991), a phenomenon that is explained by an opponent process model (Bolger, DeLongis, Kessler, \& Schilling, 1989; Solomon, 1980). In short, this theory states that intense reactions are followed by intense opposing reactions. The observed heightened mood reactivity to positive events in MDD may find its origin in such opponent processes.

In the depressed group, observed changes in mood states over the day are suggestive of a diurnal pattern with more severe symptoms in the morning, as has been previously described (American Psychiatric Association, 1994). Mood reactivity was largely unaffected by this diurnal pattern, as only decreases in NA following positive events were slightly attenuated over the course of the day.

Although mood responses to daily events were small in magnitude, the results provide important information about the daily lives of clinically depressed individuals. The relatively strong response to positive events in participants with MDD may explain why behavioral activation in itself, intended to increase the frequency and enjoyability of pleasant events, is an effective treatment for MDD (Jacobson et al., 1996). Positive events may help change the attentional and behavioral focus of depressed individuals (Watkins \& Teasdale, 2001) and act as a buffer against the damaging effects of neuroendocrine abnormalities associated with stress (McEwen, 1998). Our results suggest that engagement in positive events may pose a complicated challenge for depressed people, as they tend to appraise them as relatively stressful. In addition to an increase in PA, stressful positive events lead to an additional increase in NA in depressed people. This can be understood in light of symptoms such as feeling insecure, fatigue, diminished ability to think or concentrate, and psychomotor retardation. Two examples of positive events in the same depressed woman may illustrate this point. In the first example, drinking coffee with the partner was rated as rather pleasant (5), rather important (5), and not stressful (1). Having breakfast with all family members was rated as rather pleasant (5) and highly important (6) but at the same time moderately stressful (4). Although both events were considered pleasant and important, the second event appears to have been more challenging. This may help clarify why it is important in behavioral therapy for MDD to introduce more complex and strenuous activities in daily life only after an initial improvement in clinical condition (Beck et al., 1979).

Significant effects of clinical characteristics in MDD on reactivity of mood states were all related to negative events. Of particular interest is the finding of greater NA reactivity in depressed participants with a positive family history. This may point toward a heritable component in mood reactivity to daily hassles, which has also been found in the reactivity of the HPA axis (Holsboer, Lauer, Schreiber, \& Krieg, 1995).

A few limitations of the study should be noted. With respect to the clinical assessments, all SCID interviews were administered by the same person; reliability of the diagnosis of MDD therefore cannot be directly evaluated. However, given that the full SCID was administered by an experienced psychiatrist in a specialized affective disorder outpatient clinic and that severity of the current episode was confirmed by HDRS and BDI data, it seems improbable that many patients were misdiagnosed. Although information about previous episodes and family history of mood disorders was obtained systematically, false negatives cannot be ruled out; any underreporting of these clinical variables could lead to underestimation of their effects on mood reactivity.

Sample characteristics place some constraints on generalization of results. The current MDD sample consisted of individuals presenting for outpatient treatment at a mental health care facility, and results may not necessarily apply to depressed people in the community or in other treatment settings. The healthy comparison group differed from the MDD group in educational level and in employment status (owing mainly to MDD-related work disability) - sociodemographic variables that potentially influence exposure to different kinds of daily events. Their actual influence cannot be determined. However, the absence of significant weekend versus weekday effects suggests that work-related differences in daily activity patterns or stressors are unlikely to explain the differences in mood reactivity in the two groups. Similarly, mood reactivity results were essentially unchanged after the addition of educational level as a predictor in the multilevel model.

Mood and event data may also have been vulnerable to certain sources of error. Even with a time-sampling approach like ESM, retrospective bias cannot be entirely ruled out. Event assessment was based on self-reports only, as there was no practical means of verifying event occurrence. The method of event assessment makes it also impossible to clarify whether similar events were classified differently (either positive or negative) by depressed and healthy participants. This may have affected the comparison of event frequencies between the groups. Furthermore, the causal association between events and mood states cannot be firmly established, as event and mood reports were collected at the same points in time. Current mood levels could, for example, influence the probability of reporting positive or negative events in the preceding interval. The finding that prior events, controlling for effects of current events, were associated with persistent changes in both mood states does support our assumption that events influenced mood and not vice versa. The multilevel model used in this analysis did not allow us to test the hypothesis that current mood alters the probability that future events will occur; this could be the case if, for example, high NA increased the probability of getting involved in an argument in the near future. Finally, there may have been inaccuracies in participants' reports of the times at which they completed the ESM forms (Litt, Cooney, \& Morse, 
1998). Salivary cortisol measures collected at the same times as the ESM measures showed clear diurnal curves (Peeters, Nicolson, \& Berkhof, 2002), suggesting that most of the participants were able to comply with the procedure most of the time. More important, we have no reason to suspect that MDD and healthy participants differed in their compliance with the protocol in any way that could have systematically biased the results.

This study contributes new information about the effects of daily events on mood states in clinically depressed individuals. Results indicate that depressed individuals responded less strongly to negative events but exhibited larger mood responses to positive events than did nondepressed individuals. Given that low PA is a key feature of mood in MDD, these findings suggest that clinical interventions focused on daily experience may be useful in elevating positive mood states. Future studies should address neuroendocrine responses associated with changes in mood states in depressed people. Finally, it is important to investigate whether mood reactivity normalizes following clinical recovery.

\section{References}

American Psychiatric Association. (1994). Diagnostic and statistical manual of mental disorders (4th ed.). Washington, DC: Author.

Beck, A. T., Rush, A. J., Shaw, B. F., \& Emery, G. (1979). Cognitive therapy of depression. New York: Guilford Press.

Beck, A. T., Ward, C. H., \& Mendelson, M. (1961). An inventory for measuring depression. Archives of General Psychiatry, 4, 561-571.

Bolger, N., DeLongis, A., Kessler, R. C., \& Schilling, E. A. (1989). Effects of daily stress on negative mood. Journal of Personality and Social Psychology, 57, 808-818.

Bolger, N., \& Zuckerman, A. (1995). A framework for studying personality in the stress process. Journal of Personality and Social Psychology, 69, 890-902.

Clark, D., Beck, A., \& Stewart, B. (1990). Cognitive specificity and positive-negative affectivity: Complementary or contradictory views on anxiety and depression? Journal of Abnormal Psychology, 99, 148-155.

Connor, T. J., \& Leonard, B. E. (1998). Depression, stress and immunological activation: The role of cytokines in depressive disorders. Life Sciences, 62, 583-606.

Coyne, J. C. (1994). Self-reported distress: Analog or Ersatz depression? Psychological Bulletin, 116, 29-45.

Coyne, J. C., Aldwin, C., \& Lazarus, R. S. (1981). Depression and coping in stressful episodes. Journal of Abnormal Psychology, 90, 439-447.

Coyne, J. C., Gallo, S. M., Klinkman, M. S., \& Clarco, M. M. (1998). Effects of recent and past major depression and distress on self-concept and coping. Journal of Abnormal Psychology, 107, 86-96.

Csikszentmihalyi, M., \& Larson, R. (1987). Validity and reliability of the experience-sampling method. Journal of Nervous and Mental Disease, $175,526-536$.

David, J. P., Green, P. J., Martin, R., \& Suls, J. (1997). Differential roles of neuroticism, extraversion, and event desirability for mood in daily life: An integrative model of top-down and bottom-up influences. Journal of Personality and Social Psychology, 73, 149-159.

Delespaul, P. (1995). Assessing schizophrenia in daily life: The experience sampling method. Maastricht, the Netherlands: University Press Maastricht

Derogatis, L., Lipman, R., \& Cori, L. (1973). SCL-90, an outpatient rating scale. Preliminary report. Psychopharmacology Bulletin, 9, 542-575.

deVries, M. (Ed.). (1992). The experience of psychopathology: Investigating mental disorders in their natural settings. Cambridge, England: Cambridge University Press.

Diener, E., Colvin, C. R., Pavot, W. G., \& Allman, A. (1991). The psychic costs of intense positive affect. Journal of Personality and Social Psychology, 61, 492-503.

Diener, E., \& Emmons, R. A. (1984). The independence of positive and negative affect. Journal of Personality and Social Psychology, 47, $1105-1117$

First, M. B., Spitzer, R. L., Gibbon, M., \& Williams, J. B. W. (1995) Structured Clinical Interview for DSM-IV Axis I Disorders. New York: Biometrics Research Department, New York State Psychiatric Institute.

Flett, G. L., Vredenburg, K., \& Krames, L. (1997). The continuity of depression in clinical and nonclinical samples. Psychological Bulletin $121,395-416$.

Gable, S. L., Reis, H. T., \& Elliot, A. J. (2000). Behavioral activation and inhibition in everyday life. Journal of Personality and Social Psychology, 78, 1135-1149.

Goldstein, H., Rasbash, J., Plewis, I., Draper, D., Browne, W., Yang, M., et al. (1998). A user's guide to MLwiN. London: Multilevel Models Project, Institute of Education, University of London.

Grosscup, S. J., \& Lewinsohn, P. M. (1980). Unpleasant and pleasant events, and mood. Journal of Clinical Psychology, 36, 252-259.

Hamilton, M. (1967). Development of a rating scale for primary depressive illness. British Journal of Social and Clinical Psychology, 6, 278-296.

Holahan, C. J., Moos, R. H., Holahan, C. K., \& Cronkite, R. C. (2000). Long-term posttreatment functioning among patients with unipolar depression: An integrative model. Journal of Consulting and Clinical Psychology, 68, 226-232.

Holsboer, F. (2000). The corticosteroid receptor hypothesis of depression. Neuropsychopharmacology, 23, 477-501.

Holsboer, F., Lauer, C. J., Schreiber, W., \& Krieg, J. C. (1995). Altered hypothalamic-pituitary-adrenocortical regulation in healthy subjects at high familial risk for affective disorders. Neuroendocrinology, 62, 340347.

Jacobson, N. S., Dobson, K. S., Truax, P. A., Addis, M. E., Koerner, K., Gollan, J. K., et al. (1996). A component analysis of cognitivebehavioral treatment for depression. Journal of Consulting and Clinical Psychology, 64, 295-304.

Jolly, J. B., Murray, J., Kramer, T. A., \& Wherry, J. N. (1994). Integration of positive and negative affectivity and cognitive content-specificity: Improved discrimination of anxious and depressive symptoms. Journal of Abnormal Psychology, 103, 544-552.

Kanner, A. D., Coyne, J. C., Schaefer, C., \& Lazarus, R. S. (1981). Comparison of two modes of stress measurement: Daily hassles and uplifts versus major life events. Journal of Behavioral Medicine, 4, (1), $1-39$.

Kendler, K. S., Thornton, L. M., \& Gardner, C. O. (2000). Stressful life events and previous episodes in the etiology of major depression in women: An evaluation of the "kindling" hypothesis. American Journal of Psychiatry, 157, 1243-1251.

Krieg, J. C., Lauer, C. J., Schreiber, W., Modell, S., \& Holsboer, F. (2001). Neuroendocrine, polysomnographic and psychometric observations in healthy subjects at high familial risk for affective disorders: The current state of the "Munich Vulnerability Study." Journal of Affective Disorders, 62, (1-2), 33-37.

Langston, C. A. (1994). Capitalizing on and coping with daily-life events: Expressive responses to positive events. Journal of Personality and Social Psychology, 67, 1112-1125.

Lazarus, R. S., \& Folkman, S. (1984). Stress, appraisal, and coping. New York: Springer.

Lewinsohn, P. M. (1975). The behavioral study and treatment of depression. In M. Hersen, R. M. Eisler, \& P. M. Miller (Eds.), Progress in behavior modification, (Vol. 1, pp. 19-64). New York: Academic Press.

Lewinsohn, P. M., \& Amenson, C. S. (1978). Some relations between pleasant and unpleasant mood-related events and depression. Journal of Abnormal Psychology, 87, 644-654.

Litt, M., Cooney, N., \& Morse, P. (1998). Ecological momentary asses- 
ment (EMA) with treated alcoholics: Methodological problems and potential solutions. Health Psychology, 17, 48-52.

Maier, W., Lichtermann, D., Minges, J., \& Heun, R. (1992). Personality traits in subjects at risk for unipolar major depression: A family study perspective. Journal of Affective Disorders, 24, 153-163.

Marco, C. A., Neale, J. M., Schwartz, J. E., Shiffman, S., \& Stone, A. A. (1999). Coping with daily events and short-term mood changes: An unexpected failure to observe effects of coping. Journal of Consulting and Clinical Psychology, 67, 755-764.

McEwen, B. S. (1998). Protective and damaging effects of stress mediators. New England Journal of Medicine, 338(3), 171-179.

Morris, W. N. (1999). The mood system. In D. Kahneman, E. Diener, \& N. Schwarz (Eds.), Well-being: The foundations of hedonic psychology, (pp. 169-189). New York: Russell Sage Foundation.

Peeters, F. P. M. L., Nicolson, N. A., \& Berkhof, J. (2002). Levels and variability of daily life cortisol secretion in major depression. Manuscript submitted for publication.

Ravindran, A. V., Griffiths, J., Waddell, C., \& Anisman, H. (1995). Stressful life events and coping styles in relation to dysthymia and major depressive disorder: Variations associated with alleviation of symptoms following pharmacotherapy. Progress in Neuro-Psychopharmacology \& Biological Psychiatry, 19, 637-653.

Santor, D. A., \& Coyne, J. C. (2001). Evaluating the continuity of symptomatology between depressed and nondepressed individuals. Journal of Abnormal Psychology, 110, 216-225.

Segal, Z. V., Williams, J. M., Teasdale, J. D., \& Gemar, M. (1996). A cognitive science perspective on kindling and episode sensitization in recurrent affective disorder. Psychological Medicine, 26, 371-380.

Segerstrom, S. C., Taylor, S. E., Kemeny, M. E., \& Fahey, J. L. (1998), Optimism is associated with mood, coping, and immune change in response to stress. Journal of Personality and Social Psychology, 74, $1646-1655$.

Solomon, R. L. (1980). The opponent-process theory of acquired motiva- tion: The costs of pleasure and the benefits of pain. American Psychologist, 35, 691-712.

Suls, J., Green, P., \& Hillis, S. (1998). Emotional reactivity to everyday problems, affective inertia, and neuroticism. Personality and Social Psychology Bulletin, 24, 127-136.

Swendsen, J. D. (1998). The helplessness-hopelessness theory and daily mood experience: An idiographic and cross-situational perspective. Journal of Personality and Social Psychology, 74, 1398-1408.

Swendsen, J. D., Tennen, H., Carney, M. A., Affleck, G., Willard, A., \& Hromi, A. (2000). Mood and alcohol consumption: An experience sampling test of the self-medication hypothesis. Journal of Abnormal Psychology, 109, 198-204.

Taylor, S. E. (1991). Asymmetrical effects of positive and negative events: The mobilization-minimization hypothesis. Psychological Bulletin, 110, $67-85$.

van Eck, M., Berkhof, H., Nicolson, N., \& Sulon, J. (1996). The effects of perceived stress, traits, mood states, and stressful daily events on salivary cortisol. Psychosomatic Medicine, 58, 447-458.

van Eck, M., Nicolson, N. A., \& Berkhof, J. (1998). Effects of stressful daily events on mood states: Relationship to global perceived stress. Journal of Personality and Social Psychology, 75, 1572-1585.

Watkins, E., \& Teasdale, J. D. (2001). Rumination and overgeneral memory in depression: Effects of self-focus and analytic thinking. Journal of Abnormal Psychology, 110, 353-357.

Watson, D. (2000). Mood and temperament. New York: Guilford Press. Watson, D., Clark, L., Weber, K., Assenheimer, J. S., Strauss, M., \& McCormick, R. (1995). Testing a tripartite model: II. Exploring the symptom structure of anxiety and depression in student, adult, and patient samples. Journal of Abnormal Psychology, 104, 15-25.

Received August 14, 2001

Revision received May 2, 2002

Accepted July 24, 2002 